\title{
The masquerades of pelvic tuberculosis: A case of early invasive adenocarcinoma with multiple pelvic lymphadenopathy
}

Department of Obstetrics and Gynecology, Catholic University Medical College, Seoul, Korea

Su Jeong Lee, Ja Hyun Koo, Seong Hye Park, Jeong Namkoong, Keun Ho Lee,

Tae Chul Park, Jong Sup Park, Chan Joo Kim

\section{Case Report}

The incidence of adenocarcinoma of the uterine cervix is increasing. Microinvasive adenocarcinoma of the cervix has been thought to behave more aggressive than its microinvasive squamous cell carcinoma of the cervix counterpart.

We report a case of a 42-year-old woman with screening cytology 'HSIL' and cervical conization exhibiting microinvasive cervical adenocarcinoma. (Figure 1)

Figure 1. a) Pap smear : HSIL (x40) b) and c)Punch Biopsy shows adenoClS (blue) and CIN2(red circle ), (x100, x40)

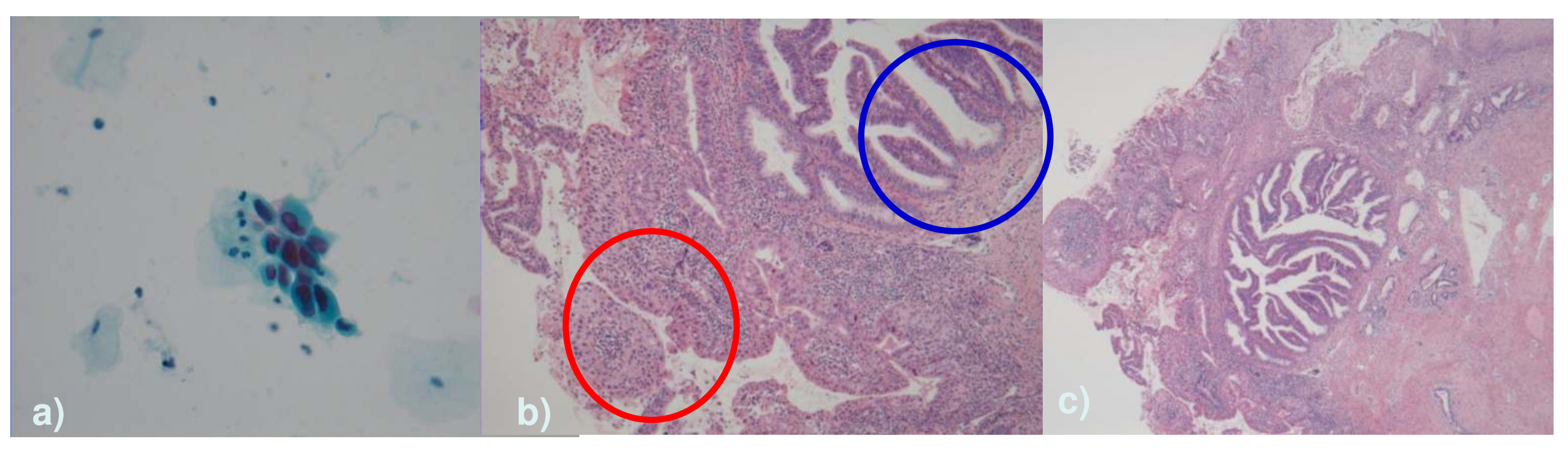

Based on the histological result of endocervical portion with adenoCIS, a modified radical hysterectomy was performed.

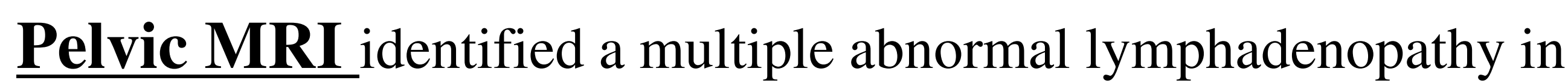
right external and internal iliac chains with no discernible cervical tumor mass. (Figure 2)

Figure 2. T2 weighted pelvic MRI (2014.8.29)

a) No definite discernible mass is noted in uterine cervix

b) Multiple abnormal LAPs in right external and internal iliac chains

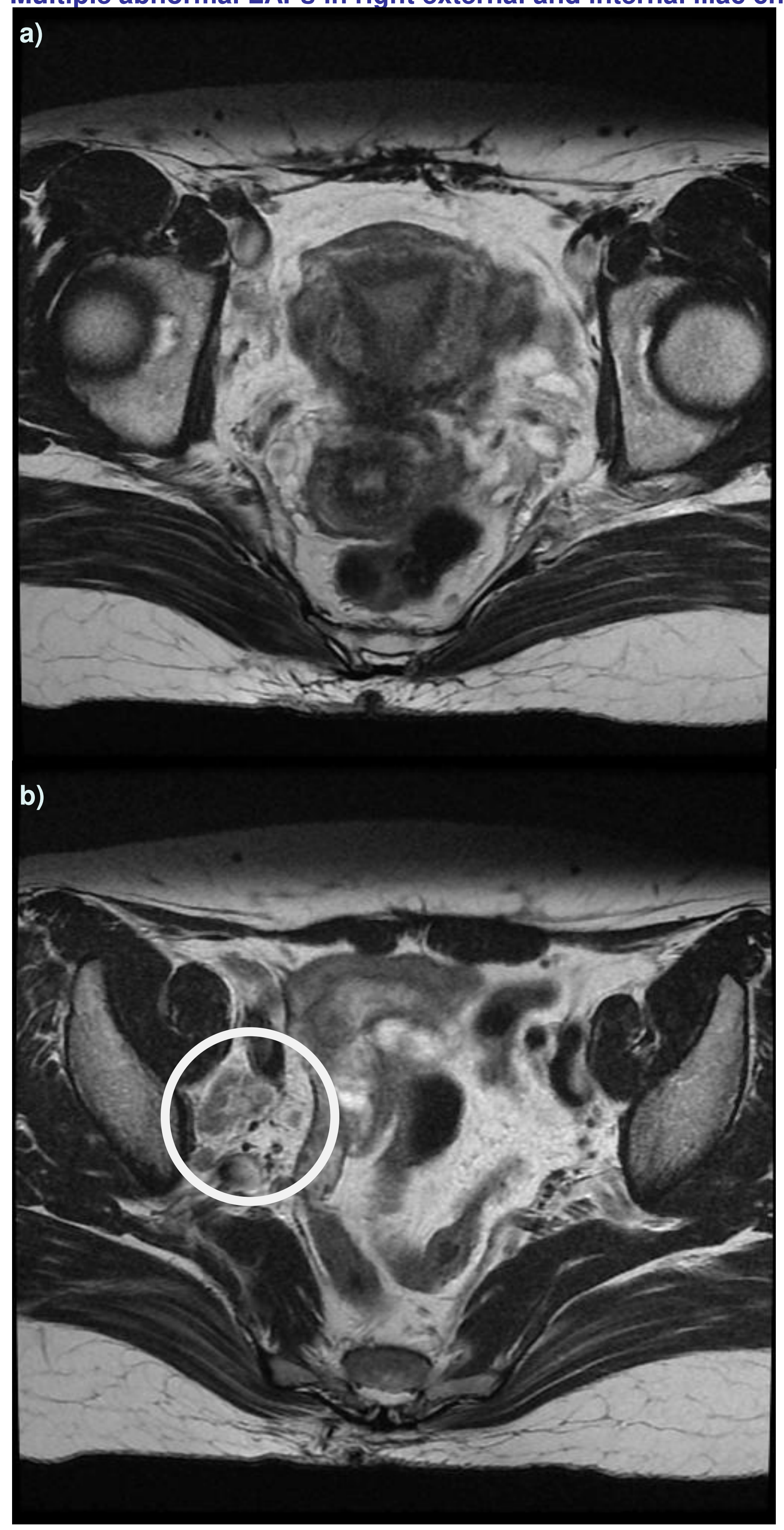

Figure 3. Chronic granuloma with multinucleated giant cells

a) Microinvasive adenocarcinoma (100X), b) Microinvasive adenocarcinoma (200X)

c) Chronic granuloma (LN 40X), d) Chronic granuloma (LN 100X)

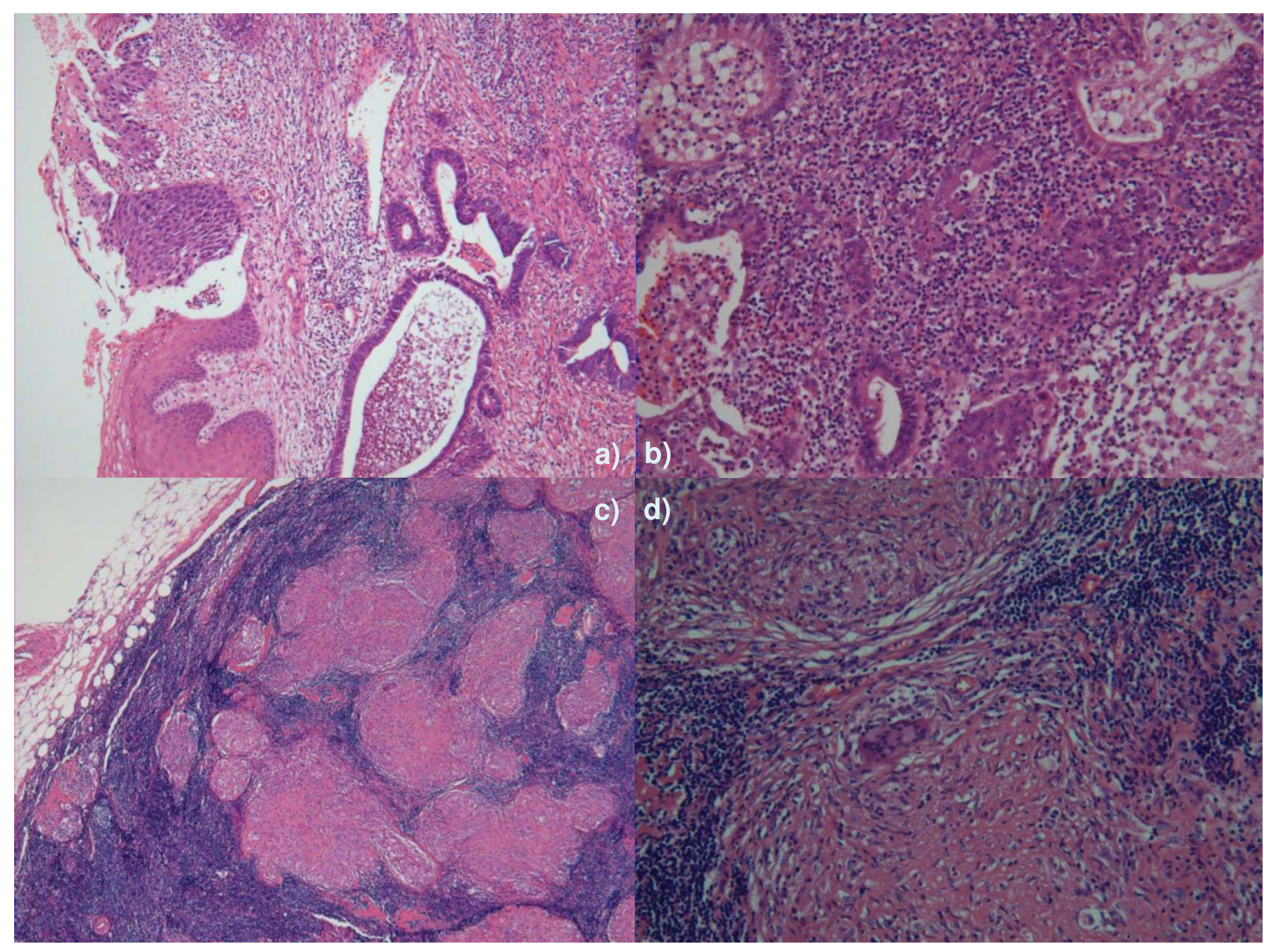

Radical hysterectomy with Rt adnexectomy with pelvic LN dissection was done in $17^{\text {th }}$,september, 2014.

During operation, excision of abnormal enlarged right external iliac and common iliac lymph node was done. Frozen section of those demonstrated chronic granuloma. Findings of lymph node was consistent with tuberculosis including chronic granulomas with multinucleated giant cells. (Figure 3)

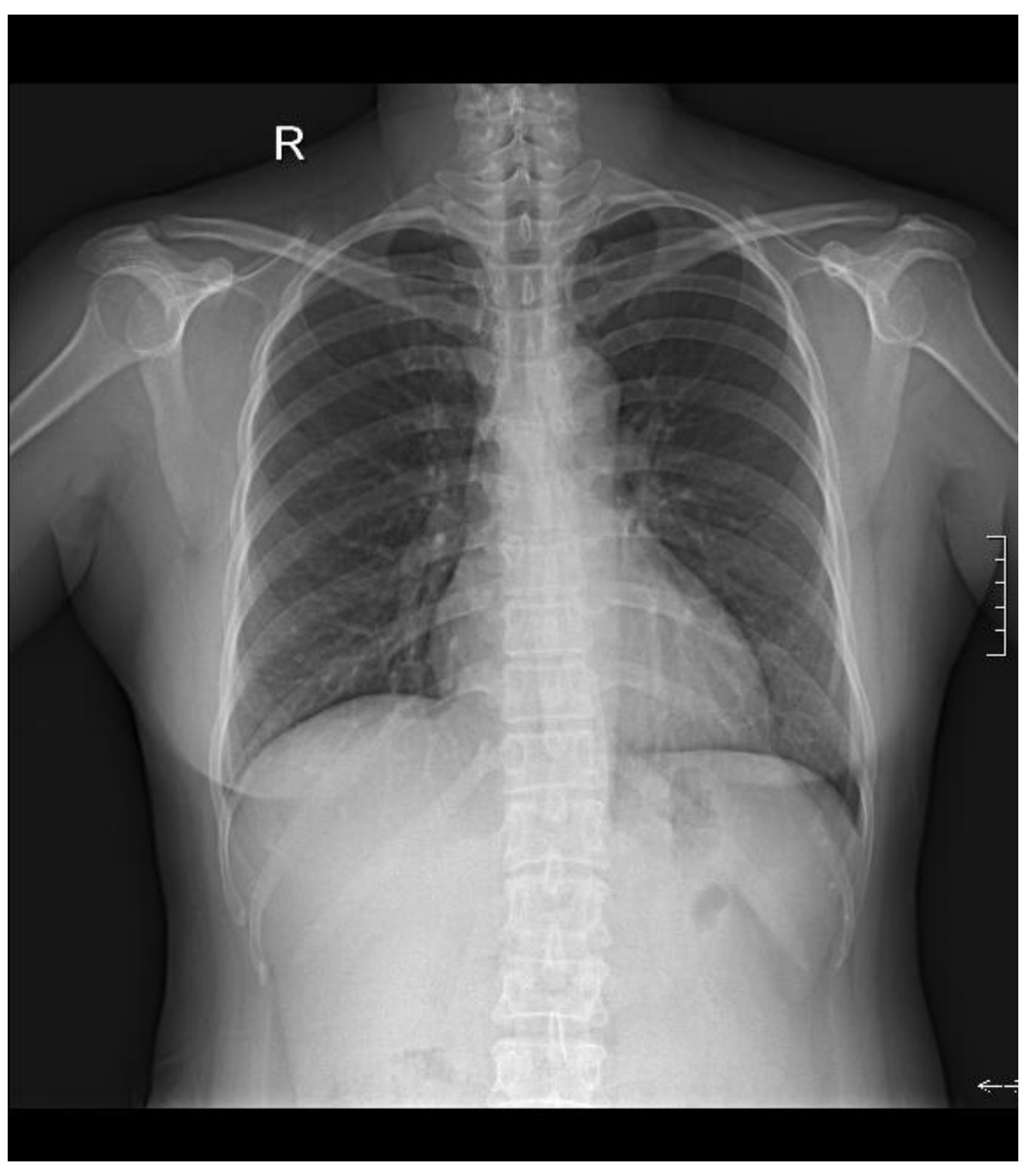

Figure 4. Chest PA shows thymoma ( $2 \times 3 \mathrm{~cm})$ with no Tuberculosis lesion. She underwent VATS thymoma excision on $13^{\text {th }}$, october, 2014.

After 7 month tuberculosis medication with isoniazid, rifampicin, ethambutol and pyrazinamide, the patient continued follow-up evaluation for 38 month.

Findings of the multiple pelvic lymphadenopathy in cervical adenocarinoma is a rare case and also important because it is poorly symptomatic and potentially it would be harmful if missed the proper time of treatment. 